\title{
Diagnostics in anaemia of chronic disease in general practice: a real-world retrospective cohort study
}

\author{
Annemarie Schop, Msc ${ }^{1 *}$, Karlijn Stouten, $\mathrm{PhD}^{2}$, Ron van Houten, $\mathrm{MD}^{3}$, \\ Jürgen Riedl, $\mathrm{PhD}^{4}$, Joost van Rosmalen, $\mathrm{PhD}^{5}$, Patrick JE Bindels, MD, PhD ${ }^{6}$, \\ Mark-David Levin, MD, PhD ${ }^{7}$
}

${ }^{1} \mathrm{PhD}$ Student, Department of Internal Medicine, Albert Schweitzer Hospital, Dordrecht, The Netherlands; ${ }^{2}$ Clinical Chemist Resident, Department of Clinical Chemistry, Albert Schweitzer Hospital, Dordrecht, The Netherlands; ${ }^{3} \mathrm{GP}$, General Medical Practice Van Houten, Hendrik-Ido-Ambacht, The Netherlands; ${ }^{4}$ Clinical Chemist, Department of Clinical Chemistry, Albert Schweitzer Hospital, Dordrecht, The Netherlands; ${ }^{5}$ Assistant Professor of Biostatistics, Department of Biostatistics,

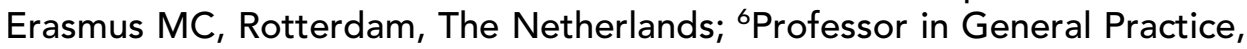
Department of General Practice, Erasmus MC, Rotterdam, The Netherlands; ${ }^{7}$ Internist-Hematologist, Department of Internal Medicine, Albert Schweitzer Hospital, Dordrecht, The Netherlands
*For correspondence: a.schop2@ asz.nl

Competing interests: The authors declare that no competing interests exist.

Received: 20 November 2017 Accepted: 14 March 2018 Published: 25 July 2018

(c) This article is Open Access: CC BY license (https:// creativecommons.org/licenses/ by/4.0/)

Author Keywords: anaemia of chronic disease, inflammation anaemia, general practice, primary care, diagnosis

Copyright (c) 2018, The Authors; DOI:10.3399/

bjgpopen18X101597

\section{Abstract}

Background: Limited research has been performed that focused on the diagnosis of the underlying cause of anaemia of chronic disease (ACD) in general practice or on prevalence data of the underlying causes of ACD in general practice, although this is one of the most common types of anaemia.

Aim: To clarify the diagnostic strategies of GPs in patients newly diagnosed with ACD and to determine the most common underlying causes.

Design \& setting: Retrospective cohort study.

Method: Patients newly diagnosed with ACD were selected based on laboratory criteria. ACD was defined as confirmed anaemia and ferritin levels above $100 \mu \mathrm{g} / \mathrm{l}$ combined with decreased iron and/ or reduced transferrin. Additional medical information on patients was obtained from the electronic medical files of the GP and/or the referral hospital.

Results: Of the 267 analysed patients with ACD, additional investigations were performed in 205 patients (77\%); in 31 patients (12\%) the cause was apparent at the time of diagnosis, and for 31 patients (12\%) no additional investigations were requested. In 210 (79\%) of the 267 patients, an underlying cause was established, with infection $(n=68,32 \%)$, autoimmune disease $(n=51,24 \%)$ and malignancy $(n=48,23 \%)$ as the most frequently observed etiologies. In $35(13 \%)$ of the ACD patients, oral iron supplementation was prescribed by the GP. This was mainly done in patients with severe anaemia or less enhanced ferritin levels.

Conclusion: For most patients with newly diagnosed ACD, the GP undertakes additional investigations to establish underlying causes. However, the cause of ACD remains unknown in a small proportion of patients. The use of oral iron supplementation in these patients requires caution. 


\section{How this fits in}

This study provides an overview of the most commonly requested additional investigations by GPs and presents the prevalence of the most common underlying causes of ACD in general practice. $A C D$ is often observed in general practice. Establishing the underlying cause of $A C D$ is essential, since this condition can lead to reduced quality of life and/or increased risks related to possibly detrimental underlying diseases. Finally, this study demonstrates that $13 \%$ of ACD patients still receive oral iron supplementation, mainly those patients with severe anaemia and less enhanced ferritin levels.

\section{Introduction}

Anaemia is a common finding in general practice and is associated with increased mortality, physical and cognitive decline, collapse, fractures, frailty, cardiovascular events, and reduced quality of life. ${ }^{1,2}$ ACD is one of the most common types of anaemia. ${ }^{3-6}$ This type of anaemia can be caused by a variety of conditions, including acute and chronic infections, chronic diseases, autoimmune disorders, acute trauma, surgical interventions, renal failure, heart failure, and malignancies. ${ }^{7-13}$

$A C D$ is described as a functional iron deficiency caused by elevated hepcidin levels, which implies that oral iron supplementation is unnecessary. ${ }^{14-16}$ Patients with $A C D$ aged $\geq 50$ years have a relative risk for mortality of 1.48 compared to adults without anaemia. ${ }^{17}$ To ensure proper treatment of $A C D$, the underlying cause needs to be elucidated and treated. If the cause is not clear, additional investigations are required. $2,5,15,16,18,19$ These steps require active participation of the GPs involved with patients with ACD in general practice.

The National Institute for Health and Care Excellence (NICE) published guidelines on the management and treatment of anaemia in patients with chronic kidney disease, which is an underlying cause often observed in ACD patients. ${ }^{20}$ Besides these guidelines, there is not much research available focusing specifically on the diagnosis of the underlying cause of ACD in general practice, or on prevalence data of the underlying causes of ACD in general practice. Therefore, this study aims to clarify the diagnostic strategies employed by GPs for their patients with ACD and provides an overview of the underlying causes as established by GPs and/or medical specialists.

\section{Method}

\section{Patient inclusion}

Patients were recruited as part of a transmural project aimed at anaemic patients aged $\geq 50$ years. The main goal of the project is to improve the care for anaemic patients, and it has been running since $2007 .^{21}$

In patients presenting to one of the 63 participating GPs with symptoms indicative of anaemia, an extensive laboratory protocol was performed that consisted of haemoglobin, mean corpuscular volume, C-reactive protein and/or erythrocyte sedimentation rate, vitamin B12, creatinine, ferritin, folic acid, lactate dehydrogenase, transferrin, reticulocytes, leukocytes, thrombocytes, and serum iron. ${ }^{18}$ Anaemia was defined as haemoglobin $\leq 13.7 \mathrm{~g} / \mathrm{dL}(8.5 \mathrm{mmol} / \mathrm{L})$ for males and $\leq 12.1 \mathrm{~g} / \mathrm{dL}$ $(7.5 \mathrm{mmol} / \mathrm{L})$ for females. ACD was defined as confirmed anaemia and ferritin $\geq 100 \mu \mathrm{g} / \mathrm{l} \mathrm{combined}$ with decreased serum iron $(<14 \mu \mathrm{mol} / \mathrm{L}$ for males and $<10 \mu \mathrm{mol} / \mathrm{L}$ for females) and/or reduced transferrin (below the lower limit of the reference interval). ${ }^{21,22}$ As part of the transmural project, a comment was added to the laboratory results alerting GPs to the most likely cause of anaemia.

For the present study, patients diagnosed with ACD were retrospectively selected from the large database that was compiled between 1 February 2007 and 1 February 2013.

\section{Data collection}

Clinical data was extracted from the electronic medical files of the GPs and referral hospital. Data could only be collected on the basis of the willingness of the GP to participate in the study and the availability of the data in electronic documentation. If these conditions could not be met, the patient was excluded from the study $(n=564)$ (Figure 1).

Since patients were recruited over a 6-year period, the length of follow-up varied considerably. The end of follow-up was recorded as the date of data collection, or the date of the last available 


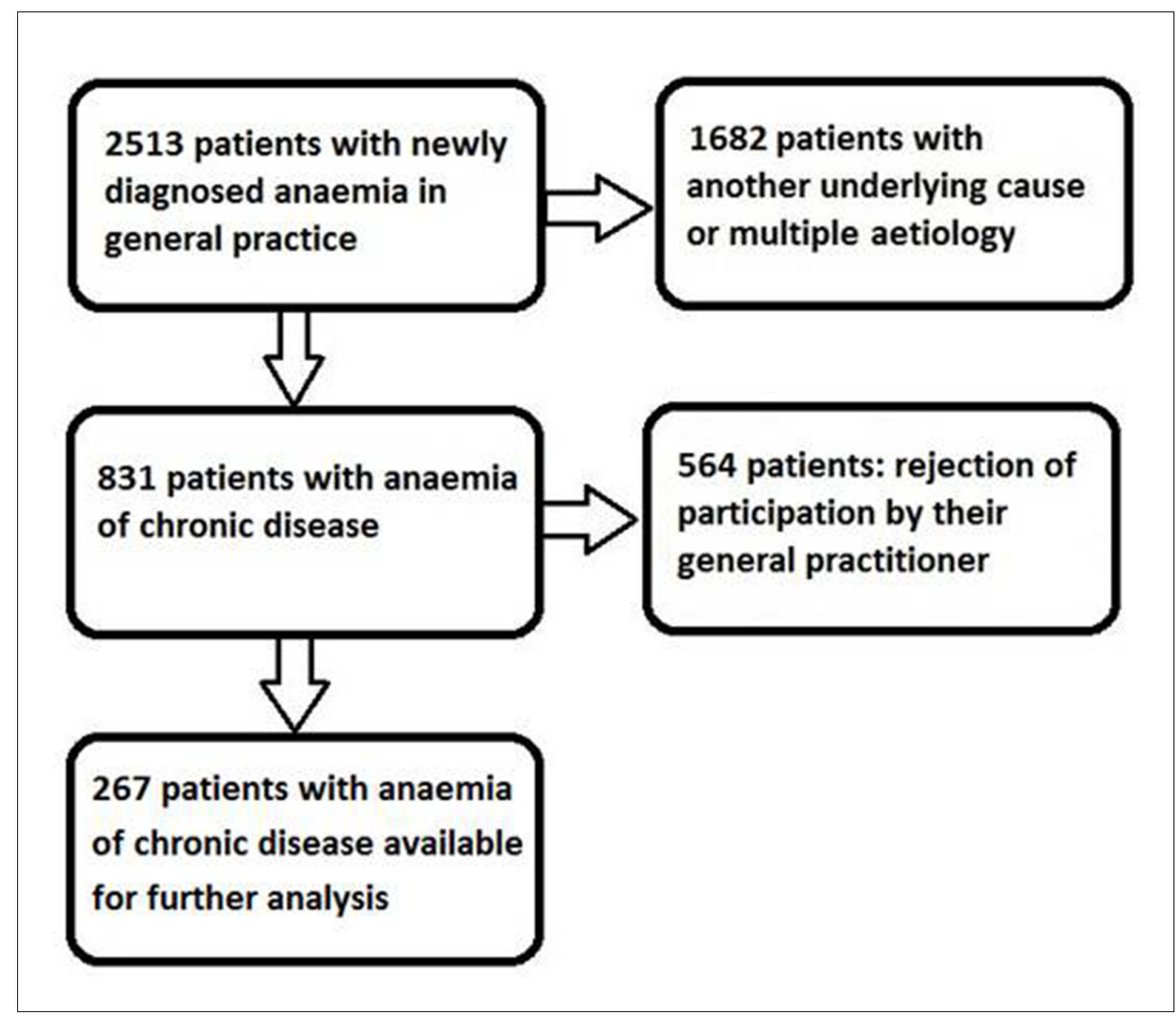

Figure 1. Flow diagram of the selection of study patients.

documented data (due to patients changing their GP in case of relocation or migration). If the patient had died before the moment of data collection, the date of death was recorded as the end of follow-up.

Collected clinical information consisted of all diagnostic investigations ordered by GPs, including those requested in consultation with a medical specialist, from the moment of discovery of ACD until the diagnosis of the underlying cause or the first outpatient visit to the referral hospital. These investigations were defined as 'additional investigations'. In addition, all underlying causes of ACD (those diagnosed by the GP and those diagnosed by the referral hospital's medical specialist) were registered. If the patient was known to suffer from diabetes, chronic lung disease, or heart failure, and no other underlying cause of ACD could be established, these diseases were assigned as the underlying cause of $A C D .^{7-13}$ Any prescription of oral iron supplementation, as well as the time (in weeks) from the confirmation of ACD to the establishment of the underlying cause were also recorded.

\section{Definitions}

Guidelines published by the Dutch College of General Practitioners in 2003 (revised in 2014) recommend handling newly diagnosed ACD in general practice as follows:

1. further diagnostic investigations to clarify the underlying illness (unless an underlying cause is already known); and

2. no oral iron supplementation. ${ }^{16}$

In the present study this was defined as 'current recommendations'. 


\section{Statistical analysis}

The study population was characterised by standard descriptive statistics. Comparisons of the included and excluded patients were performed using the $\chi^{2}$ test and independent samples $t$-test (as appropriate). Differences in mean haemoglobin levels between patients referred and not referred to a medical specialist after establishment of ACD were tested using an independent samples $t$-test. Factors associated with the prescription of oral iron supplementation were analysed using the $\chi^{2}$ test, independent samples $t$-test, and multivariable logistic regression analysis. The independent variables in the logistic regression were age, sex, haemoglobin, and ferritin. Haemoglobin and ferritin were dichotomised according to the median of those variables. In addition, the same univariable and multivariable analyses were used to investigate factors associated with the recommended diagnostic and therapeutic strategy of patients with ACD.

A two-sided $P$-value $\leq 0.05$ was considered statistically significant. Data were analysed using the SPSS for Windows (version 24).

\section{Results}

\section{Patient characteristics}

Between 1 February 2007 and 1 February 2013, patients with newly diagnosed anaemia were included in a large transmural project. ${ }^{21}$ In 831 (33\%) of these 2513 patients, the anaemia was defined as ACD based on predefined laboratory criteria and evaluated by experienced clinical chemists. All tests were performed in the clinical chemistry laboratory of the Albert Schweitzer Hospital.

Because of lack of consent of the GP due to time restraints and loss of follow-up, a total of 267 patients (32\%) with newly diagnosed ACD were finally available for the analysis (Figure 1). Characteristics of the study group are presented in Table 1.

No significant difference was found between the included patients and the non-participants regarding sex $(P=0.11)$, but a small difference in age was found (mean age of included patients 74.3 years versus 73.5 years in non-participants, $P=0.05$ ). For both males and females, no significant differences in haemoglobin, ferritin, or transferrin levels were found between the included patients and non-participants (data not shown). In addition, the included patients demonstrated a significantly lower serum iron level compared with the non-participants $(P=0.003$ for males, $P=0.01$ for females).

Table 1. Characteristics of the study population $(n=267)$.

\begin{tabular}{lcc}
\hline Sex & Mean \pm SD & Reference value \\
\hline Age, years & 148 male & \\
\hline Male & $74.3 \pm 10.5$ & \\
\hline Female & $72.3 \pm 10.7$ & \\
\hline Haemoglobin, g/dl & $76.7 \pm 9.7$ & $13.7-17.7$ \\
\hline Male & $11.8 \pm 1.3$ & $12.1-16.1$ \\
\hline Female & $12.4 \pm 1.1$ & $2.0-3.6$ \\
\hline Transferrin, g/l & $11.0 \pm 1.0$ & $25-250$ \\
\hline Ferritin, $\mu \mathrm{g} / \mathrm{l}$ & $1.9 \pm 0.4$ & $20-150$ \\
\hline Male & & \\
\hline Female & $445 \pm 325$ & $14-28$ \\
\hline Serum iron, $\mu \mathrm{mol} / \mathrm{l}$ & $379 \pm 373$ & $10-25$ \\
\hline Male & & \\
\hline Female & $7.1 \pm 3.7$ & \\
\hline
\end{tabular}




\section{Additional investigations by GPs}

In 205 (77\%) of the 267 included patients with newly established ACD, additional investigations were requested by GPs to clarify the underlying illness (Table 2). Moreover, in 78 patients multiple investigations were performed, resulting in a total of 311 investigations. The most frequently requested additional investigations were chest X-ray (24\%), referral to an internist $(21 \%)$, and referral to the emergency room (10\%). The cause was already apparent at the time of diagnosing the ACD in 31 patients (12\%), mostly due to diabetes mellitus (39\%) and autoimmune diseases (32\%). For 31 patients (12\%), no additional investigations were requested, even though the cause of ACD was not clear. Patients who were referred to a medical specialist ( $n=145,54 \%)$, either at initial diagnosis or after additional investigations by the GP, had a mean haemoglobin level of 11.7 (range 8.1-13.5) g/ dl compared with 11.8 (range 7.9-13.5) $\mathrm{g} / \mathrm{dl}$ in patients who were not referred $(P=0.378)$.

\section{Underlying causes of ACD}

In $210(79 \%)$ patients an underlying cause of ACD was determined, most frequently infection ( $n=$ $68,32 \%)$, autoimmune disease ( $n=51,24 \%)$, and malignancy ( $n=48,23 \%)$ (Table 3$)$.

In 154 patients (58\%), the median time from presentation with anaemia to clarification of the underlying cause could be collected; this was 2 weeks (range 0-48). Of these 154 patients, the underlying cause was established within 1 week for 66 (43\%), and within 4 weeks after presentation for 103 patients (67\%). Further information is available from the authors on request.

\section{Current guideline recommendations}

In 205 (77\%) patients, the GP acted according to the current Dutch guidelines (additional investigations to clarify the underlying illness causing ACD [unless an underlying cause was already known], and no oral iron supplementation prescribed). These recommendations were adhered to more

Table 2. Data on established cause and recorded investigations

\begin{tabular}{lc} 
& Frequency, $\mathbf{n}(\%)$ \\
\hline Physical examination ${ }^{\mathrm{a}}$ & $17(5.5)$ \\
\hline X-ray & $75(24.1)$ \\
\hline Chest & $11(3.5)$ \\
\hline Abdomen & $9(2.9)$ \\
\hline Joint & $2(0.6)$ \\
\hline Sinus & $28(9.0)$ \\
\hline Ultrasound, abdomen & $12(3.9)$ \\
\hline CT scan, abdomen/thorax ${ }^{\mathrm{b}}$ & \\
\hline Endoscopy & $2(0.6)$ \\
\hline Full endoscopy & $5(1.6)$ \\
\hline Gastroscopy & $5(1.6)$ \\
\hline Colonoscopy & \\
\hline Referral & $66(21.2)$ \\
\hline Internist & $32(10.3)$ \\
\hline Emergency room & $12(3.9)$ \\
\hline Pulmonologist & $11(3.5)$ \\
\hline Geriatrician & $8(2.6)$ \\
\hline Rheumatologist & $16(5.1)$ \\
\hline Other & 311 \\
\hline Total & \\
\hline a & \\
\hline
\end{tabular}

${ }^{a}$ Only physical examinations directly related to a diagnosis of the underlying cause were noted. ${ }^{\mathrm{b}} \mathrm{CT}$ scan was always requested in consultation with a medical specialist. CT= computed tomography. 
Table 3. Underlying causes of anaemia of chronic disease

\begin{tabular}{|c|c|c|c|c|}
\hline & $\begin{array}{c}\text { Additional } \\
\text { investigations, } n(\%) \\
(n=205 ; 77 \%)\end{array}$ & $\begin{array}{l}\text { Underlying disease already } \\
\text { apparent, } n(\%) \\
(n=31 ; 12 \%)\end{array}$ & $\begin{array}{l}\text { No additional investigation, no apparent } \\
\text { cause, } n(\%) \\
(n=31 ; 12 \%)\end{array}$ & $\begin{array}{l}\text { Total, } n(\%) \\
(n=267 ; \\
100 \%)\end{array}$ \\
\hline $\begin{array}{l}\text { Underlying cause } \\
\text { established }\end{array}$ & $179(87)$ & $31(100)$ & $0(0)$ & $210(79)$ \\
\hline Autoimmune disease & $41(23)$ & $10(32)$ & - & $51(24)$ \\
\hline Infection & $68(38)$ & - & - & $68(32)$ \\
\hline Renal failure & $4(2)$ & - & - & $4(2)$ \\
\hline Recent operation & $2(1)$ & $1(3)$ & - & $3(1)$ \\
\hline Malignancy & $44(25)$ & $4(13)$ & - & $48(23)$ \\
\hline Diabetes & $7(4)$ & $12(39)$ & - & $19(9)$ \\
\hline Heart failure & $5(3)$ & $2(7)$ & - & $7(3)$ \\
\hline Chronic lung disease & $4(2)$ & $1(3)$ & - & $5(2)$ \\
\hline Other causes ${ }^{a}$ & $4(2)$ & $1(3)$ & - & $5(2)$ \\
\hline No cause established & $26(13)$ & $0(0)$ & $31(100)$ & $57(21)$ \\
\hline
\end{tabular}

Percentage of underlying causes is calculated from the total number of causes established in each subgroup. ${ }^{a}$ Such as liver cirrhosis, haematoma, and alcohol abuse.

frequently in patients with a high ferritin level, that is $>324 \mu \mathrm{g} / \mathrm{l}$ (OR 2.46 ; $95 \%$ confidence interval $[\mathrm{Cl}]=1.33$ to 4.52), as shown in Table 4. In addition, less frequent implementation of the recommendations in older patients (OR $0.97,95 \% \mathrm{Cl}=0.94$ to 1.00 ) and patients with more severe anaemia, that is haemoglobin $<11.8 \mathrm{~g} / \mathrm{dl}(\mathrm{OR} 0.47,95 \% \mathrm{Cl}=0.2$ to 1.03$)$ was detected, but these associations were not statistically significant.

\section{Oral iron supplementation}

Of all 267 patients, 35 (13\%) received oral iron supplementation despite being diagnosed with ACD and demonstrating ferritin levels $\geq 100 \mu \mathrm{g} / \mathrm{l}$. Severe anaemia (haemoglobin $<11.8 \mathrm{~g} / \mathrm{dl}$ ) and less

Table 4. Factors associated with implementation of the current recommendations and prescription of oral iron supplementation

Univariate analysis

Percentage or mean difference $(95 \% \mathrm{Cl})$

Recommendations

Age, years

3.63 (0.65 to 6.60)

Female

Male

Haemoglobin $\geq 11.8 \mathrm{~g} / \mathrm{dl}$

Haemoglobin $<11.8 \mathrm{~g} / \mathrm{dl}$

Ferritin $>324 \mu \mathrm{g} /$

Ferritin $\leq 324 \mu \mathrm{g} / \mathrm{l}$

Oral iron supplementation

Age, years

$-1.29(-5.05$ to 2.47$)$

15.1

Female

Male

Haemoglobin $\geq 11.8 \mathrm{~g} / \mathrm{dl}$

Haemoglobin $<11.8 \mathrm{~g} / \mathrm{dl}$

Ferritin $>324 \mu \mathrm{g} / \mathrm{l}$

Ferritin $\leq 324 \mu \mathrm{g} / \mathrm{l}$

$\mathrm{Cl}=$ confidence interval
Multivariate analysis

Odds ratio $(95 \% \mathrm{Cl})$

$0.97(0.94$ to 1.00$)$

Reference category

$0.82(0.38$ to 1.75$)$

Reference category

$0.47(0.21$ to 1.03$)$

Reference category

2.46 (1.33 to 4.52$)$

1.00 (0.97 to 1.04$)$

Reference category 2.00 (0.83 to 4.83)

Reference category

4.97 (1.82 to 13.62$)$

Reference category

2.30 (1.06 to 4.99$)$ 
enhanced ferritin levels $(<324 \mu \mathrm{g} / \mathrm{l})$ were associated with a higher probability of oral iron supplementation (OR $4.97 ; 95 \% \mathrm{Cl}=1.82$ to 13.62 and $\mathrm{OR} 2.30 ; 95 \% \mathrm{Cl}=1.06$ to 4.99 , respectively), as shown in Table 4.

\section{Discussion}

\section{Summary}

This study provides a representative overview of the diagnostic strategies employed by GPs for their patients with ACD and an overview of the underlying causes as established by GPs and/or medical specialists. Additional investigations were requested in $77 \%$ of the analysed 267 ACD patients. The most frequently requested additional investigations were chest $x$-ray (24\%), referral to an internist $(21 \%)$, and referral to the emergency room (10\%). The underlying cause of ACD was established in $79 \%$ of these patients, with the most common causes being infection (in $32 \%$ ), autoimmune disease (in 24\%), and malignancy (in 23\%). The median time from presentation with anaemia to clarification of the underlying cause of $A C D$ was 2 weeks. The prescription of oral iron supplementation in patients with $A C D$ is not recommended and requires caution. ${ }^{14-16}$

\section{Strengths and limitations}

A possible limitation of this study is related to the recruitment of patients. Of all GPs participating in the project, about one-third declined participation (mainly due to time constraints). However, the group of newly diagnosed ACD patients not included in this study presented no significant difference in sex, or haemoglobin, ferritin, or transferrin levels compared with those included in the analyses. In addition, because a difference in age and in serum iron levels was observed between the groups, all analyses were corrected for these two variables. Another limitation is the retrospective design of the study, which can lead to differences in the completeness of individual GPs' medical reports and, perhaps, to loss of patient information, such as the reason to decline additional investigations after establishment of ACD.

Strengths of the study include a large sample size of representative patients providing a realistic overview of patients with ACD in general practice. In addition, patients were selected based on well-defined laboratory criteria, making these results generalisable to other countries. Finally, the content of the Dutch guidelines used for handling newly diagnosed patients with ACD is also widely accepted in other guidelines worldwide..$^{2,20,23}$

\section{Comparison with existing literature}

This study demonstrated that $77 \%$ of the ACD patients received additional investigations after establishment of the ACD, with the most common strategy being referral to a medical specialist (47\%). This relatively large proportion of referrals might be because the diagnosis or treatment of the underlying cause of ACD might be beyond the scope of GPs' focus, for example, the diagnosis or treatment of malignancies. In addition, for $12 \%$ of patients without a clear cause of ACD, no additional investigations were requested. For most of these patients, no reason was documented for disregarding further investigations. However, GPs may have considered these patients too vulnerable for extensive tests due to, for example, advanced age and/or comorbidities, or the lack of consequences in these frail patients. ${ }^{24}$

Comparable studies analysing the underlying causes of ACD have investigated hospitalised populations, whereas no data are available for community-dwelling adults with ACD. In this study, the most common underlying causes of ACD were infections, autoimmune inflammation, and malignancies; this is in line with data from hospitalised patients with ACD. ${ }^{4,25,26}$ However, compared with the present study population, a markedly higher prevalence of renal insufficiency was reported among hospitalised patients with ACD. ${ }^{3,25}$ This might be due to selection bias in the present study population; the transmural project separated renal anaemia as a distinct category of anemia, resulting in less renal insufficiency in this ACD patient group. In addition, since only patients with newly diagnosed anaemia (that is, no anaemia in the preceding 2 years) were included, this probably excluded patients frequently hospitalised and those already being treated by, for instance, a nephrologist.

In this study, the diagnostic strategy of GPs was in accordance with the current Dutch recommendations in $77 \%$ of the patients with newly diagnosed ACD. Patients presenting with high ferritin 
levels (>324 $\mu \mathrm{g} / \mathrm{l}$ ) were more often treated according to these recommendations. Since increased ferritin is a diagnostic parameter for ACD, elevated levels of ferritin might be an alarm symptom for GPs, prompting them to more frequently perform additional investigations. In addition, more increased ferritin levels might exclude iron deficiency more clearly, thereby preventing unnecessary oral iron supplementation. A trend was observed towards less frequent implementation of the recommendations in older patients and patients with more severe anaemia. In older patients, multiple diseases and/or comorbidities often determine the clinical presentation (that is, health status). Therefore, especially in these patients, GPs might decide to forgo additional investigations. Patients with severe anaemia may be rapidly treated with oral iron supplementation to elevate haemoglobin levels. However, this is not recommended in patients with ACD.

Nevertheless, about $13 \%$ of the patients in this study still received oral iron supplementation as part of their treatment, even though no benefit is expected due to enhanced levels of hepcidin and, therefore, reduced oral iron absorption. ${ }^{2,14-16}$ In this study, severe anaemia and minimally reduced ferritin levels (i.e. $<324 \mu \mathrm{g} / \mathrm{l}$ ) are associated with increased oral iron prescription by GPs. The association of minimally reduced ferritin levels and the more frequent prescription of oral iron supplementation might be caused by the absence of a definite ferritin cut-off value defining iron deficiency. 16,21 The effect of oral iron supplementation in patients with ACD might cause unnecessary side effects and drug-drug interactions, and can lead to unnecessary (although small) costs. ${ }^{27,28}$

\section{Implications for practice}

To the authors' knowledge, this is the first study to explore and describe additional investigations to establish underlying causes in patients with newly diagnosed ACD in primary care. In these patients, infections, autoimmune diseases, and malignancies were the most frequently occurring underlying causes. Oral iron supplementation, which is not indicated in ACD patients, needs to be abandoned in primary care to prevent unnecessary side effects and costs, as well as possible drug-drug interactions. The information emerging from this study and data on the prevalence of the underlying causes in primary care may lead to more targeted investigations and, potentially, to a more targeted diagnosis and more appropriate treatment.

\section{Funding}

This research received a grant from the ORAS fundation and Friends of the Albert Schweitzer Hospital foundation.

\section{Ethical approval}

The protocol of this retrospective cohort study was approved by the local Medical Ethics Committee of the Albert Schweitzer Hospital (trial number: 2014.51) and was conducted in accordance with the Declaration of Helsinki. The content of the database was approved by the internal ethics committee of the Albert Schweitzer Hospital, Dordrecht, the Netherlands.

\section{Provenance}

Freely submitted; externally peer reviewed.

\section{Acknowledgements}

The authors thank the participating GPs for their generous contribution to this study.

\section{References}

1. Lipschitz D. Medical and functional consequences of anemia in the elderly. J Am Geriatr Soc 2003; 51(3 Suppl): 10-13. doi: 10.1046/j.1532-5415.51.3s.6.x

2. Cavill I, Auerbach M, Bailie GR, et al. Iron and the anaemia of chronic disease: a review and strategic recommendations. Curr Med Res Opin 2006; 22(4): 731-737. doi: 10.1185/030079906X100096

3. Patel KV. Epidemiology of anemia in older adults. Semin Hematol 2008; 45(4): 210-217. doi: 10.1053/j. seminhematol.2008.06.006

4. Weiss G, Goodnough LT. Anemia of chronic disease. N Engl J Med 2005; 352(10): 1011-1023. doi: 10.1056/ NEJMra041809

5. Weiss G. Anemia of chronic disorders: new diagnostic tools and new treatment strategies. Seminars Hematol 2015; 52(4): 313-320. doi: 10.1053/j.seminhematol.2015.07.004

6. Fraenkel PG. Understanding anemia of chronic disease. Hematology Am Soc Hematol Educ Program 2015; 2015: 14-18. doi: 10.1182/asheducation-2015.1.14

7. Means RT. Recent developments in the anemia of chronic disease. Curr Hematol Rep 2003; 2(2): 116-121. 
8. Similowski T, Agustí A, MacNee W, et al. The potential impact of anaemia of chronic disease in COPD. Eur Respir J 2006; 27(2): 390-396. doi: 10.1183/09031936.06.00143704

9. Kollert F, Müller C, Tippelt A, et al. Anaemia in chronic respiratory failure. Int J Clin Pract 2011; 65(4): 479486. doi: 10.1111/j.1742-1241.2011.02631.x

10. Thomas MC, Maclsaac RJ, Tsalamandris C, et al. Unrecognized anemia in patients with diabetes: a crosssectional survey. Diabetes Care 2003; 26(4): 1164-1169. doi: 10.2337/diacare.26.4.1164

11. Ezekowitz JA, McAlister FA, Armstrong PW. Anemia is common in heart failure and is associated with poor outcomes: insights from a cohort of 12065 patients with new-onset heart failure. Circulation 2003; 107(2): 223-225. doi: 10.1161/01.CIR.0000052622.51963.FC

12. Boutou AK, Hopkinson NS, Polkey MI. Anaemia in chronic obstructive pulmonary disease: an insight into its prevalence and pathophysiology. Clin Sci (Lond) 2015; 128(5): 283-295. doi: 10.1042/CS20140344

13. Pisaniello AD, Wong DT, Kajani l, et al. Anaemia in chronic heart failure: more awareness is required. Intern Med J 2013; 43(9): 999-1004. doi: 10.1111/imj.12195

14. Kroot JJ, Tjalsma H, Fleming RE, et al. Hepcidin in human iron disorders: diagnostic implications. Clin Chem 2011; 57(12): 1650-1669. doi: 10.1373/clinchem.2009.140053

15. Cullis J. Anaemia of chronic disease Clin Med. 2013; 13(2): 193-196. doi: 10.7861/clinmedicine.13-2-193

16. Van Wijk MAM, Mel M, Muller PA, et al. NHG-werkgroep Anemie. [Dutch General Practitioners Society. Anaemia guideline] Nederlands Huisartsen Genootschap. Standaard Anemie (in Dutch). Huisarts en Wetenschap 2014; 57(10): 528-536.

17. Shavelle RM, MacKenzie R, Paculdo DR. Anemia and mortality in older persons: does the type of anemia affect survival? Int J Hematol 2012; 95(3): 248-256. doi: 10.1007/s12185-012-1007-z

18. Nemeth E, Ganz T. Anemia of inflammation. Hematol Oncol Clin North Am 2014; 28(4): 671-681. doi: 10. 1016/j.hoc.2014.04.005

19. Nairz M, Theurl I, Wolf $D$, et al. Iron deficiency or anemia of inflammation? Differential diagnosis and mechanisms of anemia of inflammation. Wien Med Wochenschr 2016; 166(13-14): 411-423. doi: 10.1007/ s10354-016-0505-7

20. National Institute for Health and Clinical Excellence. Chronic kidney disease: managing anaemia. NICE guideline [NG8]. 2015. https://www.nice.org.uk/guidance/ng8 (acccessed 11 Jul 2018).

21. Stouten K, Riedl JA, Droogendijk J, et al. Prevalence of potential underlying aetiology of macrocytic anaemia in Dutch general practice. BMC Fam Pract 2016; 17(1): 113. doi: 10.1186/s12875-016-0514-z

22. Geïntegreerd Klinisch Chemisch Laboratorium (GKCL). [What can we do for you?] Wat kunnen we voor $u$ betekenen? (in Dutch). 2018. https://resultlaboratorium.nl/professionals/ (accessed $16 \mathrm{Jul}$ 2018).

23. American Family Physician Anemia. 2016. http://www.aafp.org/afp/topicModules/viewTopicModule.htm? topicModuleld=2 (accessed $11 \mathrm{Jul}$ 2018).

24. Andrès $E$, Serraj $K$, Federici $L$, et al. Anemia in elderly patients: new insight into an old disorder. Geriatr Gerontol Int 2013; 13(3): 519-527. doi: 10.1111/ggi.12017

25. Cash JM, Sears DA. The anemia of chronic disease: spectrum of associated diseases in a series of unselected hospitalized patients. Am J Med 1989; 87(6): 638-644. doi: 10.1016/S0002-9343(89)80396-1

26. Liangos O, Wald R, O'Bell JW, et al. Epidemiology and outcomes of acute renal failure in hospitalized patients: a national survey. Clin J Am Soc Nephrol 2006; 1(1): 43-51. doi: 10.2215/CJN.00220605

27. Zorginstituut Nederland. [Ferrous fumarate] Ferrofumaraat (in Dutch). 2016. https://www. farmacotherapeutischkompas.nl/bladeren/preparaatteksten/f/ferrofumaraat (accessed $16 \mathrm{Jul}$ 2018).

28. Truven Health Analytics Inc. Iron supplements (oral route, parenteral route). Side effects. 2016. http://www. mayoclinic.org/drugs-supplements/iron-supplement-oral-route-parenteral-route/side-effects/drg-20070148 (accessed $11 \mathrm{Jul}$ 2018). 